\title{
Development of BREM Software for Bistatic Radar's Range Estimations
}

\section{Desarrollo de Software BREM para Estimaciones de zona de visibilidad de radares biestáticos}

\author{
Yunior Coya Márquez, \\ yu.coya@gmail.com \\ José Raúl Machado Fernández, \\ m4ch4do@hispavista.com
}

Recibido: 21 de noviembre 2016

Aceptado: 8 de febrero 2017

\begin{abstract}
A radar device is considered to be bistatic when the transmitter and receiver are placed on locations separated by a distance considerably greater than the distance from the target. One of the key aspects when working with radars is being able to assess the range prior to deployment. In this sense, the ZVR (Zona de Visibilidad del Radar, Radar Range) software is available in Cuba since 1993 for the estimation of the range of monostatic radars. In order to complement the functions of the ZVR, a MATLAB application, which allows the $a$ priori calculation of the coverage area, was developed by the authors for the bistatic case. The new BREM (Bistatic Range Estimation in Matlab) software has an intuitive graphical interface that allows the modification of simulation data and selection of specific geographic regions on a map from Cuban territory, while other maps may be also introduced. The tool uses the direct calculation method that requires the existence of direct visibility between the transmitter and receiver, and it will be used for evaluating and planning new bistatic radar deployments.
\end{abstract}

\section{Keywords}

Bistatic Radars, Ovals of Cassini, Radar Virtual Simulation Environment, Range Estimation.

\section{Resumen}

Un dispositivo de radar es considerado como biestático cuando el transmisor y el receptor están colocados en regiones separadas por una distancia considerablemente superior a la distancia desde el blanco. Uno de los aspectos fundamentales cuando se trabaja con radares es ser capaz de determinar la zona de visibilidad antes del despliegue. En este sentido, en Cuba se cuenta desde 1993 con la aplicación ZVR (Zona de Visibilidad del Radar) para la estimación del alcance de radares monoestáticos. Para complementar las funciones del ZVR, una aplicación en MATLAB, 
que permite el cálculo apriorístico del área de cobertura, fue desarrollada por los autores para el caso biestático. La nueva aplicación BREM (Bistatic Range Estimation in Matlab, Estimación del alcance de radar en Matlab) tiene una interfaz gráfica intuitiva que permite la modificación de los datos de simulación y la elección de zonas geográficas específicas sobre un mapa del territorio cubano, si bien otros mapas pueden ser introducidos. La herramienta usa el método de cálculo directo que requiere de la existencia de visibilidad directa entre el transmisor y el receptor, y será utilizada para la evaluación y planeamiento de nuevos despliegues biestáticos.

\section{Palabras clave}

Radares Biestáticos, Óvalos de Cassini, Ambiente Virtual de Simulación de Radares, Estimación de la Zona de Visibilidad. 


\section{INTRODUCTION}

A primary radar illuminates the surroundings, like a beacon, and it receives a fraction of the released energy after being scattered by surrounding objects. Monostatic radars, which are the most common, frequently use a single antenna for transmission and reception (1). On the other hand, the system is said to be multistatic if it gathers information by simultaneously processing signals collected from transmitters, receivers or transceivers located on positions of a considerable separation (2). When multistatic radars are used, an energy gain and a consequently increase in the area of effective visibility is achieved, among other advantages (3); all they accomplished with a substantial increase in complexity, purchase cost and complexity in the operation of the equipment.

The most common practical case of multistatic radars are the bistatic ones, which are systems that operate with a pair of antennas located in remote regions (1). The most common measurements in both monostatic and bistatic radar, are those corresponding to the amplitude (4) and Doppler effect (5-6). Some of the classic multistatic papers were published by D'Addio, Farina, Conte, \& Longo (7) and Hanle (8). Additionally, researches mixing monostatic and bistatic radar can be found in the literature (9), particularly regarding clutter measurements (10).

Besides the classic aircraft detection (11), multistatic radars are applied in the calculation of soil moisture and the height of the aerial targets (12), and studies related to GPR (Ground Penetrating Radar) (13). Also, its application is recognized in most common fields such as: the guided missiles, meteorology and oceans monitoring.

In the research environment of multistatic radars, there are three subjects which are continuously being developed, although studies of diverse nature (14-15) appear with a relatively high frequency. The first of these subjects, perhaps the most classic, has to do with the improvement in the detection and tracking of targets (16-19). The second is related to the search for better alternatives for processing and merging the contributions obtained from different receptors (20-22). The third and final, of which the present project is a part, is the creation of simulation environments or libraries for estimating multistatic radars' parameters (23-26).

\subsection{Motivation and contributions}

The ZVR software was created by Cuban specialists in 1993. The facilities that it provides for radar range calculation over Cuban territory were applied to fields such as meteorology. Unfortunately, the ZVR was only dedicated to the simulation of monostatic radars. Therefore, the authors of the current paper decided to create a new application, able to estimate the range of bistatic radars.

The new tool, which they called BREM, can estimate the visibility zone of bistatic radar transmitters and receivers placed in any two points on the national territory. Additionally, visual mechanisms were implemented to ensure the easy interpretation of results. Also a digital map with a resolution of 90 meters per grid was used, which constitutes a significant upgrade when compared to the 250 meters supported by ZVR. 
The MATLAB mathematical software was chosen for the project because of its many facilities. Particularly, MATLAB is a very useful tool when performing mathematical calculations and creating visual interfaces. In addition, there is international reference of the use of MATLAB for radar related researches (27-28).

\subsection{Structure of the paper}

After introducing the topic to be discussed in section one, the authors describe the details of the investigation in sections two, three and four. Finally, the fifth section is devoted to conclusions and the sixth to recommendations.

Section two under the name of "Materials and Methods" presents the basic theory behind the implemented simulation. Thus, the major mathematical expressions involved in the direct calculation method for the existence of visibility between two remote points are offered.

Section three, "Results", is divided into three sub-sections corresponding to the facilities provided by the BREM application. The first one illustrates the two-dimensional estimation of the radar coverage area; while the second one does the same in three dimensional. The last sub-section is devoted to the software's main application: the three-dimensional range calculation taking into account the terrain profile. Therefore, it includes considerations on visibility obstruction between the target and the transmitter and receiver systems.

Section four under the name of "Discussion" reveals the strengths of BREM while it points out the ways in which software can be improved. Also, the current implementation is compared to the previously available in Cuba, and others found in the literature.

\section{MATERIALS AND METHODS}

The visibility area of a bistatic radar is represented by a geometric figure known as ovals of Cassini (4). The ovals of Cassini represent zones of equal SNR (Signal to Noise Ratio) in the region of bistatic coverage. Therefore, the visibility zone is limited by the Cassini oval whose SNR is the minimum detectable by the receiver.

If the shape of the ovals of Cassini is to be estimated, it is first necessary to consider the bistatic constant $(\mathrm{K})$. For its calculation, the user needs to introduce the radar characteristics such as: the transmitter power $\left(\mathrm{P}_{\mathrm{T}}\right)$, the gain of the transmitter and receiver antennas $\left(\mathrm{G}_{T}, \mathrm{G}_{\mathrm{R}}\right)$, the losses of the transmitter and receiver systems $\left(\mathrm{L}_{\mathrm{T}}, \mathrm{L}_{\mathrm{R}}\right)$, the equivalent noise temperature of the receiving system $\left(\mathrm{T}_{\mathrm{S}}\right)$, the noise bandwidth of the pre-detection receptor filters $\left(B_{n}\right)$, the effective bistatic radar cross section $\left(\sigma_{B}\right)$ and the minimum signal to noise ratio required for detection $(\mathrm{S} / \mathrm{N})_{\min }$. Likewise, data on environmental conditions must be provided, such as: the propagation factor between the transmitter-target and target-receptor paths $\left(\mathrm{F}_{\mathrm{T}}, \mathrm{F}_{\mathrm{R}}\right)$ and the operating wavelength ( $\lambda$ ). Using the above data, $\mathrm{K}$ can be calculated using expression [1] (4), where $\mathrm{k}$ is the Boltzmann constant. 
Note that in expression [1], $\mathrm{K}$ is equal to the maximum product of the distances of the target relative to the transmitter $\left(R_{T}\right)$ and to the receiver $\left(R_{R}\right)$.

$$
K=\left(R_{T} R_{R}\right)_{\max }=\left(\frac{P_{T} G_{T} G_{R} \lambda^{2} \sigma_{B} F_{T}^{2} F_{R}^{2}}{(4 \pi)^{3} k T_{S} B_{n}(S / N)_{\min } L_{T} L_{R}}\right)^{1 / 2}
$$

In order to know if a target can be detected by the bistatic radar, it is necessary to estimate the SNR corresponding to the target and compare it with the minimal allowed by the system. If the SNR is greater than the minimum, BREM indicates that the target will be detected in a real bistatic system; otherwise it specifies that the target cannot be detected under the given conditions.

To calculate the target's SNR, equation [1] itself must be used. It is only necessary to isolate the minimum SNR as it is done at [2]:

$$
\frac{S}{N}=\frac{P_{T} G_{T} G_{R} \lambda^{2} \sigma_{B} F_{T}^{2} F_{R}^{2}}{(4 \pi)^{3} k T_{S} B_{n}\left(R_{T} R_{R}\right)^{2} L_{T} L_{R}}
$$

Then, the product $R_{T} R_{R}$ may be obtained. To do so, the sum $\left(R_{T}+R_{R}\right)$ is first calculated by using the time difference between the wave received directly from the transmitter and the one reflected from the target $\left(\Delta \mathrm{T}_{\mathrm{rt}}\right)$, the base line $(\mathrm{L})$ which represents the distance between receiver $\left(R_{X}\right)$ and $\left(T_{X}\right)$ transmitter, and the electromagnetic wave propagation speed $c$ (speed of light). Expression [3] shows the direct method for obtaining the sum $\left(R_{T}+R_{R}\right)$.

$$
\left(R_{R}+R_{T}\right)=c \Delta T_{r t}+L
$$

The value of $R_{T}$ can be assessed by having $\left(R_{R}+R_{T}\right)$ and $R_{R}$ if the following is applied: $R_{T}=\left(R_{T}+R_{R}\right)-R_{R}$. As the value of $\left(R_{R}+R_{T}\right)$ can be extracted from [3], it is only necessary to find $R_{R}$, which can be done through the following expression [4]:

$$
R_{R}=\frac{\left(R_{T+} R_{R}\right)^{2}-L^{2}}{2\left(R_{T}+R_{R}+L \operatorname{sen} \theta_{R}\right)}
$$

Where $\theta_{\mathrm{R}}$ is the receiver's viewing angle, which is obtained from the target's azimuth angle $\left(A_{R}\right)$, the target's elevation angle $\left(E_{R}\right)$, the azimuth angle of the angular position of the transmitter $\left(\mathrm{A}_{\mathrm{RT}}\right)$ and the elevation of the angular position of the transmitter $\left(\mathrm{E}_{\mathrm{RT}}\right)$ :

$$
\theta_{R}=-\operatorname{arsen}\left[\cos E_{R} \cos E_{R T} \cos \left(A_{R}-A_{R T}\right)+\operatorname{sen} E_{R} \operatorname{sen} E_{R T}\right]
$$


Having $\theta_{R}, R_{R}$ will be obtained using [4]. Then, $R_{T}$ can be found from the sum $\left(R_{T}+R_{R}\right)$ acquired through [3]. Finally, the target's $S N R$ is calculated with $R_{R}$ and $R_{T}$. The diagram in Fig. 1 refers to the physical representation of several of the previously related magnitudes.

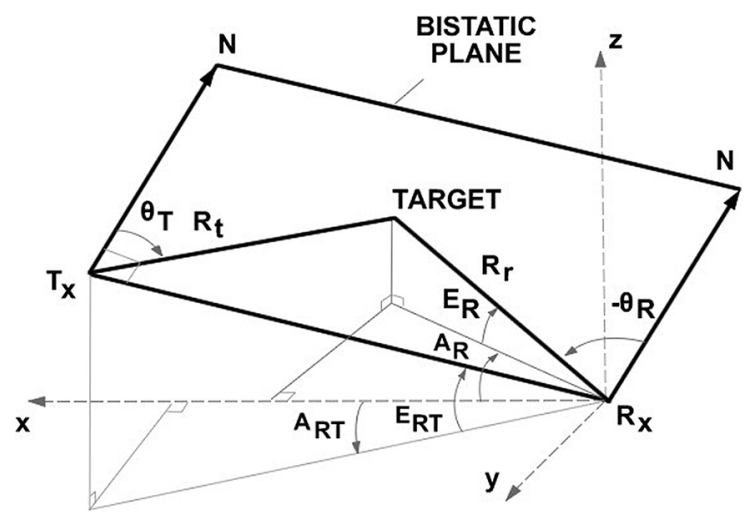

Figure 1. Geometrical relation between several radar parameters.

Finally, equations [6] and [7] are used to plot the ovals of Cassini (29). They provide the coordinates for the points that form the geometric figure, where $a=L / 2 y b=\sqrt{K}$.

$x=\left[ \pm a \sqrt{\cos 2 \theta \pm \sqrt{\left(\frac{b}{a}\right)^{4}-\operatorname{sen}^{2} 2 \theta}}\right] \cos \theta$

$$
y=\left[ \pm a \sqrt{\cos 2 \theta \pm \sqrt{\left(\frac{b}{a}\right)^{4}-\operatorname{sen}^{2} 2 \theta}}\right] \operatorname{sen} \theta
$$

The previously described procedure was the one employed by the authors to create the BREM application. The following section will describe the simulations that may be performed using the new tool.

\section{RESULTS}

Fig. 2 shows the home screen of the created application. The menu bar contains three options: Menu, Edit and Preferences. The menu option allows entering data for the 
terrain, load surface profiles and choose between the simulation modes of [1] Bistatic Plane and [2] Three Dimensions. Through the Edit button targets' information may be introduced manually or graphically. Finally, the Preferences option modifies some secondary aspects of the simulation. Note that although the software is used with maps from Cuba's ground surface, it can be adapted to any other region if the corresponding maps are properly formatted.

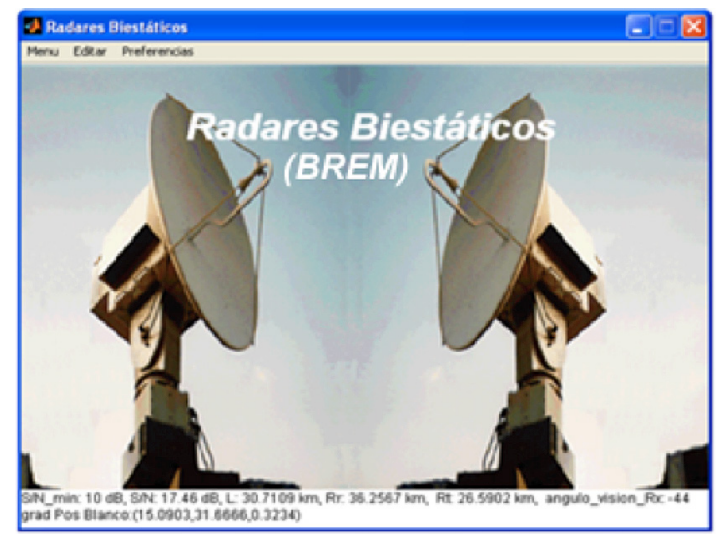

Figure 2. BREM application start screen

Next, the three main BREM features will be described. The first one shows the simulation of a bistatic plane in two dimensions; while the second one performs a three dimensional analysis. The third feature is the most important because it allows range calculations taking into account the geographic location influence.

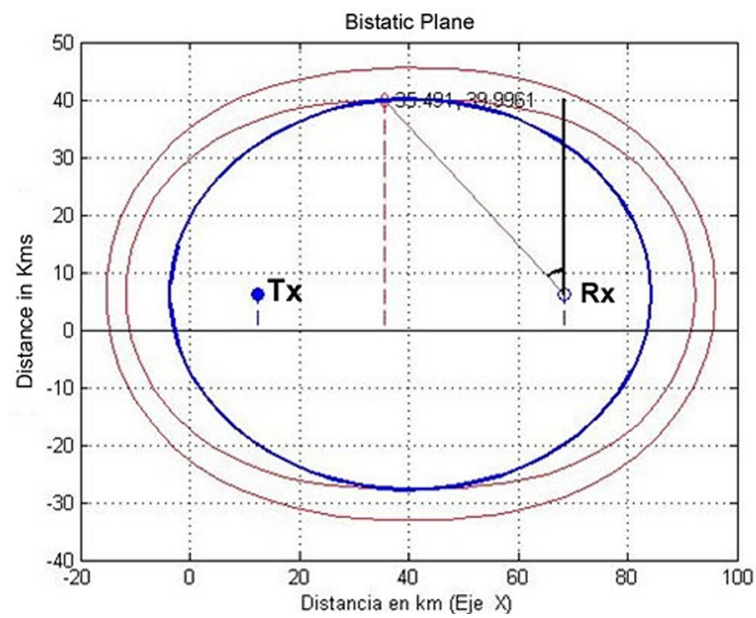

Figure 3. Calculation of the ovals of Cassini for two near points. 


\subsection{Analysis in two dimensions}

The two-dimensional analysis is the simplest simulation that can be executed with BREM. However, it is perhaps the most illustrative of the process of estimating the coverage area, because it shows the fundamental theoretical concepts used in the bistatic range estimation.

In Fig. 3, the calculation of the range, or coverage area, is shown for an example of a bistatic system where the transmitter and receiver are placed at a relatively small distance. The external line in red represents the Cassini oval with the lower SNR permitted by the system, which means that targets out of it will not be detected by the radar. The internal red line designates the oval of Cassini representing the zone of constant SNR that corresponds to the target. The blue boundary indicates the geometric place of the target's position. This geometric place is an ellipse drawn using the transmitter and receiver as focus. The sum of the distance from any point of the blue line to the transmitter with the distance between this point and the receiver is always the same.

Fig. 4 provides another example for the calculation of the bistatic radar area of visibility using a distance, between transmitter and receiver, higher than the $60 \mathrm{Km}$ used in Fig. 3 . Observe how in this case the range zone is divided into two regions, which means that there may be targets passing undetected between the transmitter and receiver. Therefore, for the same minimum SNR, if the distance between transmitter and receiver is increased, the visibility zone of the bistatic radar will decrease. It may be noted that the target obtained in Fig. 4 simulation is successfully detected by the radar system, even if it is almost at the limit of the covered area. If some of the input parameters are altered affecting the radar operation in a negative way, then BREM will indicate that the target cannot be detected.

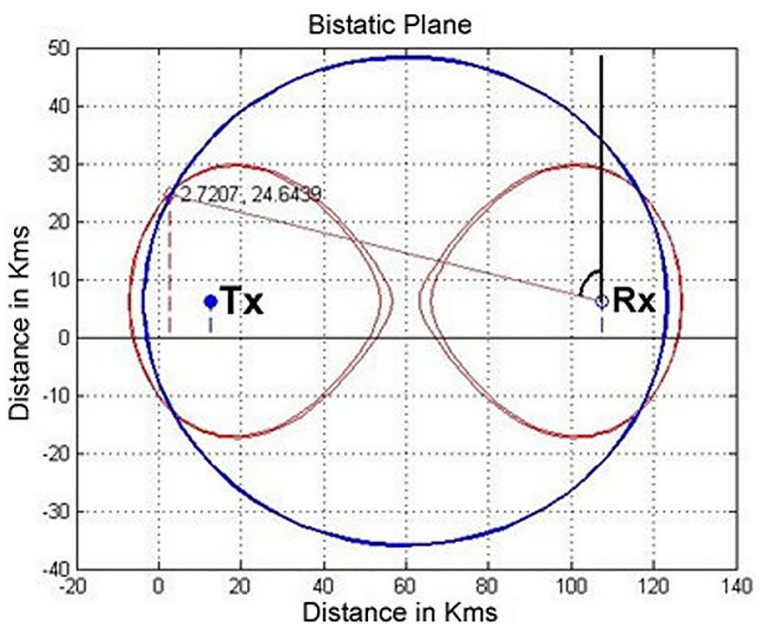

Figure 4. Calculation of the ovals of Cassini for two remote points. 


\subsection{Analysis in three dimensions}

The three-dimensional analysis is more complete than the two-dimensional one, as it provides a better understanding of the extension of the visibility zone. Fig. 5 shows an example of the computation of the bistatic range in three dimensions. The meaning of each drawn surface coincides with the explanation given for the twodimensional case.

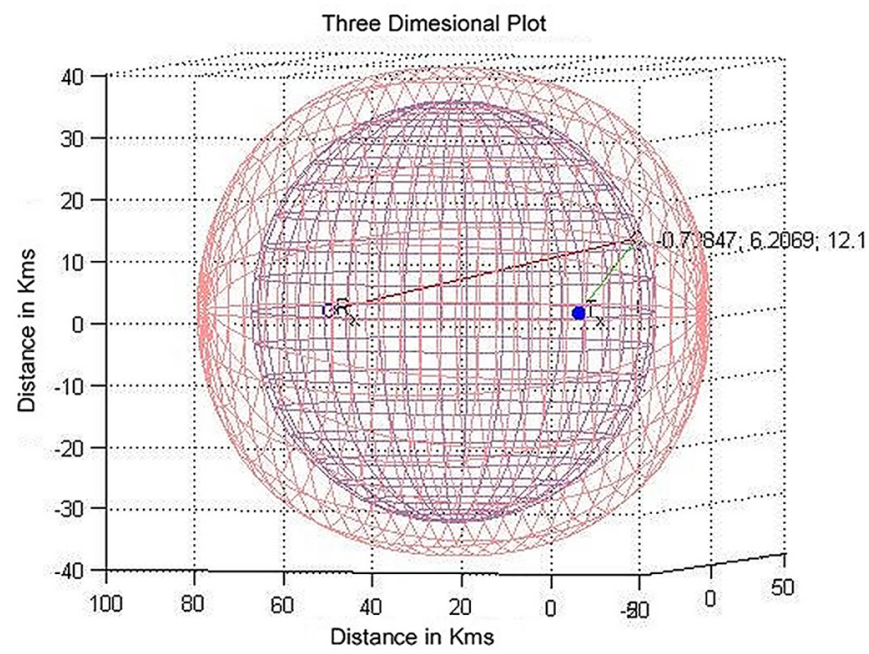

Figure 5. Analysis of bistatic radar range in three dimensions.

The GUI (Graphical User Interface) created in MATLAB allows the rotation of three-dimensional estimates obtained for achieving a better viewing from multiple angles. Fig. 6a shows an image, where both two-dimensional and three-dimensional analyses are plotted. Fig. $6 \mathrm{~b}$ shows only bistatic plane in three dimensions. These images were obtained during the development of BREM.

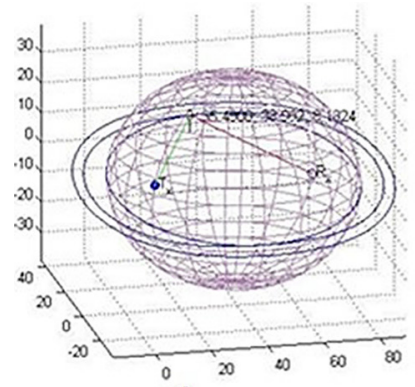

a)

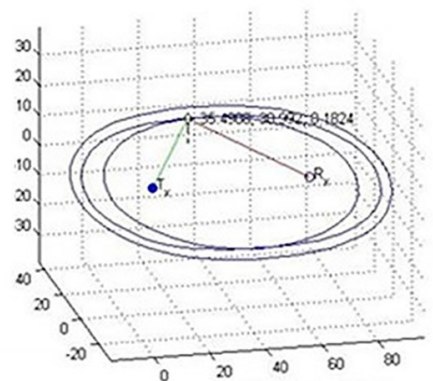

b)

Figure 6: Three-dimensional representation of the bistatic plane with and without the ellipsoid. 


\subsection{Analysis in three dimensions with surface influence}

While the two previous sections showed the potentials of BREM in the calculation of the area of visibility for bistatic radars, the most important application of the software is to perform simulations considering the terrain profile. The software allows loading a map with a resolution of 90 meters per grid from the Cuban surface and will also accept the introduction of more accurate maps.

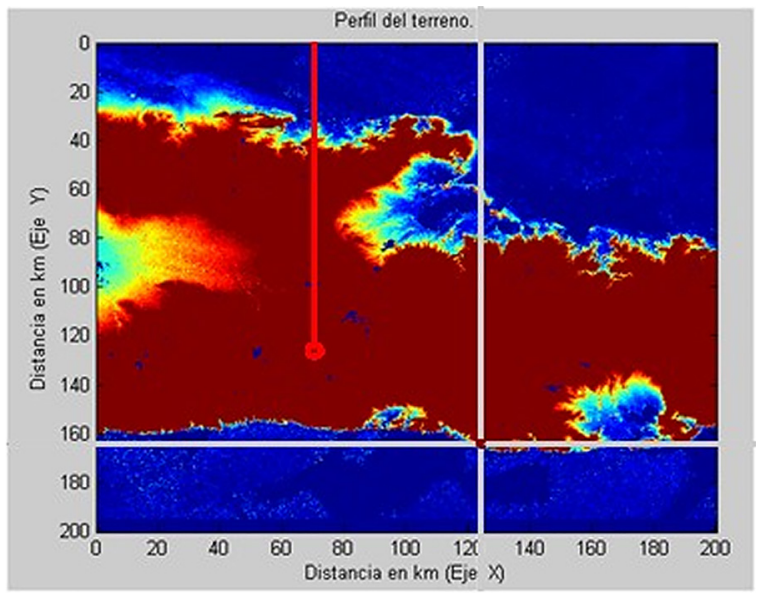

Figure.7. Selecting the simulation area.

Fig. 7 illustrates the process of selecting the simulation area in which the user will be ask to enter the points that indicate the ends of the region that may be considered during the simulation. Then, the application requests the position of the transmitter and receiver station on a map like the one shown on Fig. 8, where the terrain heights appear more in detail.

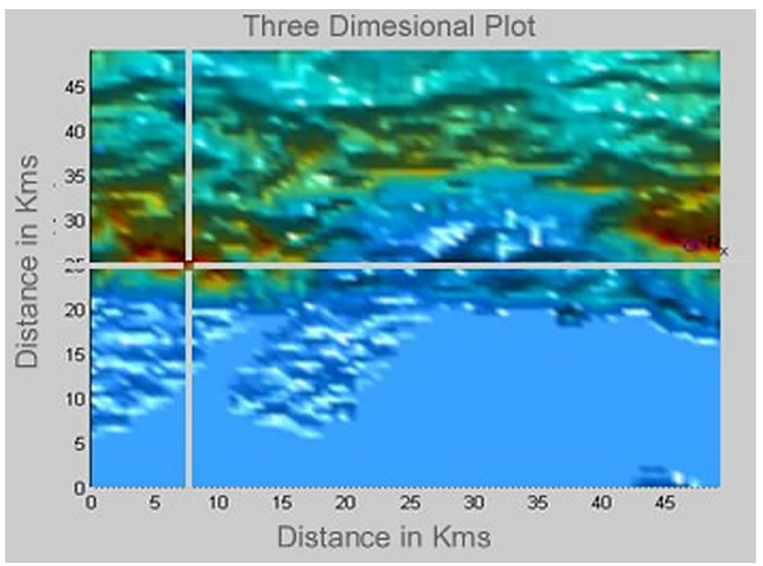

Figure 8: Selecting the transmitter and receivers' position. 
Once located the stations of the bistatic radar, the application checks that the necessary conditions for the simulation are satisfied. BREM triggers warning messages in those cases where there is no direct sight between transmitter and receiver, or between target and one of the two stations. Figures 9 and 10 show images taken from simulations.

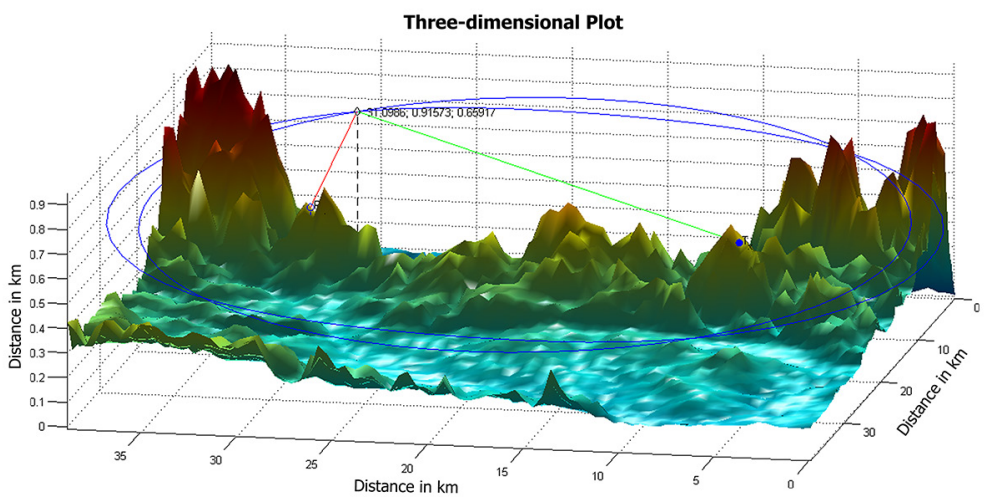

Figure 9: Estimation of the range of bistatic radars using terrain maps.

A bistatic plane and positions from a given transmitter and a given receiver are represented on figures 9 and 10. The result of the estimation of the detected target's position it's also visible. Three-dimensional calculations for the ovals of Cassini can be displayed by selecting Normal Scale (3D only) in the Preferences Menu.

\section{DISCUSSION}

The BREM application allows estimating the range of a bistatic radar whose transmitter and receiver stations are located at any point of the Cuban geography. Using the direct method that requires direct sight between transmitter and receiver, the solution complements the previously available ZVR software that provided only monostatic radar estimates.

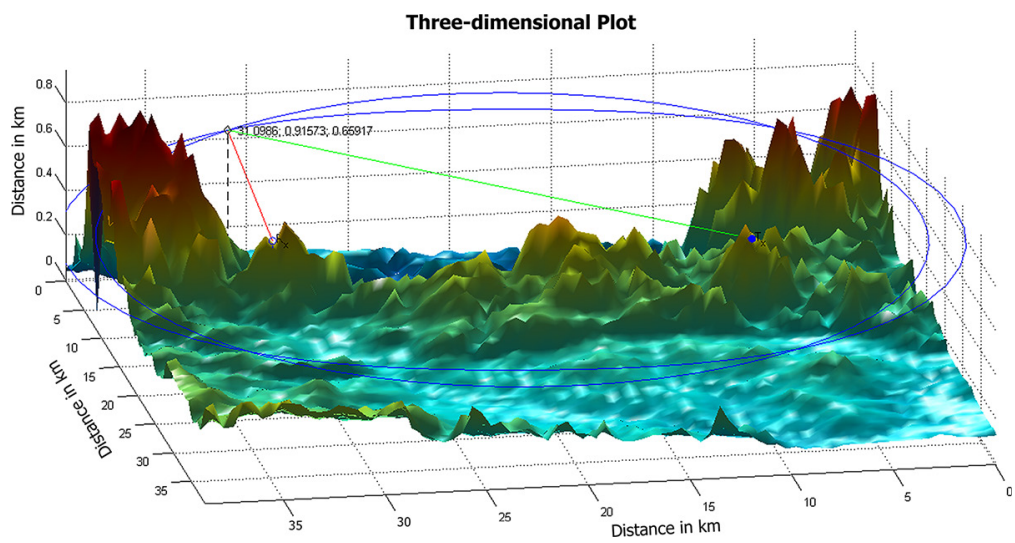

Figure 10: Rotation of Graph obtained with the BREM application. 
BREM is qualitatively superior to ZVR as it allows three-dimensional visualization of simulations, offering users an intuitive interface that allows the easy manipulation of simulation variables. Likewise, the resolution of the employed maps is better than the one used by ZVR.

The BREM software allows the introduction of data related with the receiver's perception of the target for detection analysis. The authors plan to incorporate considerations related to the Doppler effect on future developments to allow the study of moving targets situations. Similarly, it will be helpful to include the indirect calculation method for $R_{T}+R_{R}$ which enables the representation of environments where there is no direct visibility between transmitter and receiver.

BREM's estimates were validated by manual mathematical calculations performed by the authors. The authors acknowledge that the application has reduced functionalities when compared with others developed $[24,25]$ that include validation through measurements, Doppler implement considerations and include targets fluctuating amplitude. However, BREM is still in an early version and its development is conceived in a progressive way.

\section{CONCLUSIONS AND FUTURE RESEARCH}

A software named BREM was created in MATLAB 2010 for estimating the range of a bistatic radar. The solution allows the introduction of maps to simulate any desired region, while it contains maps of the Cuban terrain with a resolution of 90 meters. The main advantage of the tool is the three-dimensional visualization of the visibility zone, constructed by plotting ovals of Cassini and using the direct method for estimating the sum of the distances between target and the receiver station with target and transmitter stations. The authors will focus next on adding the simulation of fluctuating targets and Doppler effect to the software.

\section{REFERENCES}

1. Meikle, H. Modern Radar Systems (2nd Edition): Artech House; 2008. 712 p.

2. Barton, D. K., \& Leonov, S. A. Radar Technology Encyclopedia: Artech House; 1998. 536 p.

3. Bezousek, P., \& Schejbal, V. Bistatic and Multistatic Radar Systems. Radioengineering. 2008;17(3).

4. Skolnik, M. I. Radar Handbook (3er ed.): McGraw-Hill. 2008. 1328p.

5. Gungor, A. Clutter Detection in Pulse-Doppler Radar Systems. 2010.

6. Mixon, D. G. Doppler-Only Multistatic Radar. (Master of Science). Ohio: Air Force Institute of Technology, Wright-Patterson Air Force Base. 2006.

7. D’Addio, E., Farina, A., Conte, E., \& Longo, M. Multistatic Detection of Radar Signals for Swerling Models of the Target. Revista Técnica Selenia, v 9. 1985. 11-17 p.

8. Hanle, E. Survey of Bistatic and Multistatic Radar. IEE Proceedings. 1986;133(7). 587-595 p. 9. Sarabandi, K., \& Nashashibi, A. A Novel Bistatic Scattering Matrix Measurment Technique using a Monostatic Radar. IEEE Transactions on Antennas and Propagation. 1996;44(1). 41-50 p. 10. Palama, R., Greco, M., Stinco, P., \& Gini, F. Statistical Analysis of Netrad High Resolution Sea Clutter. EUSIPCO. IEEE. 2013. 1-5 p. 
11. Iannuzzelli, R. J., Schemm, C. E., \& Marcotte, F. J. Aircraft Wake Detection using Bistatic Radar: Analysis of Experimental Results. Johns Hopkins APL Technical Digest. 1998;19(3). 299 p. 12. Masters, D. S. Surface Remote Sensing Applications of GNSS Bistatic Radar: Soil Moisture and Aircraft Altimetry. [Doctor of Philosophy]. [Colorado]: Faculty of the Graduate School of the University of Colorado. 2004.

13. Counts, T., Gurbuz, A. C., Scott, W. R., McClellan, J. H., \& Kim, K. Multistatic GroundPenetrating Radar Experiments. IEEE Transactions on Geoscience and Remote Sensing. 2007;45(8). 25544-2553 p.

14. Glennon, E. P., Dempster, A. G., \& Rizos, C. Feasibility of Air Target Detection using GPS as a Bistatic Radar. Journal of Global Positioning Systems. 2006;5(1).

15. Krishnan, V., Swoboda, J., Yarman, C. E., \& Yazici, B. Multistatic Synthetic Aperture Radar Image Formation. IEEE Transactions on Image Processing. 2010;19(5). 1290-1306 p.

16. Bruyere, D. P., \& Goodman, N. A. Adaptive Detection and Diversity Order in Multistatic Radar. IEEE Transactions on Aerospace and Electronic Systems. 2008;44(4). 1615-1623 p.

17. Choi, S., Berger, C. R., Crouse, D., Willett, P., \& Zhou, S. Target Tracking for Multistatic Radar with Transmitter Uncertainty. ECE Department, University of Connecticut. 2009. 74450M-74450M-12 p.

18. Liu, W., Yilong, L., \& Fu, J. S. A Novel Threshold Optimization for Distributed OS-CFAR of Multistatic Radar Systems by Using the Genetic Algorithm. IEEE. 2001. 275-278 p.

19. Xu, S., Tang, C., Jing, P., \& Chen, Z. Efficient Centralized Track Initiation Method for Multistatic Radar. 2011. 1-7 p.

20. Efe, M., \& Soysal, G. Data Fusion in a Multistatic Radar Network using Covariance Intersection and Particle Filtering. Paper presented at the 14th International Conference on Information Fusion, Chicago. IEEE. 5-8 July 2011; Chicago, IL, USA: IEEE. 1-7 p.

21. Kay, S. Waveform Desing for Multistatic Radar Detection. Rhode Island: Dept. of Electrical, Computer, and Biomedical Engineering, University of Rhode Island. 2007; 45(3).

22. Liu, W., Lu, Y., \& Fu, J. S. CFAR Data Fusion of Multistatic Radar System under Homogeneous and Nonhomogeneous Backgrounds. The Institution of Electrical Engineers. 2002. 248-252 p.

23. Bradaric, I., Caprarp, G. T., Weiner, D. D., \& Wicks, M. C. A Framework for the Analysis of Multistatic Radar Systems with Multiple Transmitters. IEEE. 2007. 433-446 p.

24. Brooker, M. The Design and Implementation of a Simulator for Multistatic Radar Systems. [Doctor of Philosophy]. [South Africa]: University of Cape Town. 2008.

25. Ling Lim, Y. The Modelling and Simulation of Passive Bistatic Radar. [Master of Philosophy]. [Australia]: The University of Adelaide. 2013.

26. Mahdi Naghsh, M., Modarres-Hashemi, M., ShahbazPanahi, S., Soltanalian, M., \& Stoica, P. Unified Optimization Framework for Multi-Static Radar Code Design using InformationTheoretic Criteria. IEEE Transactions on Signal Processing. 2013;61(21). 5401-5416 p.

27. Kumar Yadav, A., \& Kant, L. Moving Target Detection using VI-CFAR Algorithm on MATLAB Platform. Paper presented at the International Journal of Advanced Research in Computer Science and Software Engineering. 2013;3(12) 915-918 p.

28. Santan, L. A. Análisis, estudio y simulación de los parámetros del radar en el programa MatLab. 2005.

29. Gibson, K. The Ovals of Cassini. Lecture Notes. 2007. 